\title{
TTR
}

Traduction, terminologie, re?daction

\section{L'interprétation communautaire : un modèle de communication « trialogique »}

\section{Aurelia Klimkiewicz}

Volume 18, numéro 2, 2e semestre 2005

Traduction engagée

Translation and Social Activism

URI : https://id.erudit.org/iderudit/015771ar

DOI : https://doi.org/10.7202/015771ar

Aller au sommaire du numéro

Éditeur(s)

Association canadienne de traductologie

ISSN

0835-8443 (imprimé)

1708-2188 (numérique)

Découvrir la revue

Citer cet article

Klimkiewicz, A. (2005). L'interprétation communautaire : un modèle de communication « trialogique ». TTR, 18(2), 209-224.

https://doi.org/10.7202/015771ar
Résumé de l'article

Il sera question de réfléchir sur l’interprétation communautaire, particulièrement sur les rapports qui existent entre le professionnel, le client et l'interprète lors d'un échange interculturel. Asymétrique, ce type d'intervention génère plusieurs niveaux de difficultés (langue, culture, code moral, pouvoir). En se servant du concept de tiers de Mikhaïl Bakhtine, il s'agira d'introduire une discussion sur les facteurs humains qui entrent en jeu lors d'une entrevue, au moment où l'interprète risque d'obéir à sa conscience ou à ses sentiments et non pas aux normes professionnelles et sociales.

Mots-clés: interprétation communautaire, communication interculturelle, tiers, «trialogue ».
Tous droits réservés (C) TTR: traduction, terminologie, rédaction — Les auteurs, 2007 cent protégé par la loi sur le droit d'auteur. L'utilisation des services d'Érudit (y compris la reproduction) est assujettie à sa politique d'utilisation que vous pouvez consulter en ligne.

https://apropos.erudit.org/fr/usagers/politique-dutilisation/ 


\section{L'interprétation communautaire : un modèle de communication " trialogique "}

\section{Aurelia Klimkiewicz}

Normalement la différence entre la traduction et l'interprétation est définie en termes de médium (écrit/oral), ainsi qu'en fonction de la situation de communication: en traduction celle-ci se réalise en l'absence de l'auteur et du récepteur, alors qu'en interprétation tous les participants sont réellement présents lors de l'échange verbal. Si la traduction couvre différents domaines et langues de spécialité, l'interprétation se définit davantage en fonction des contraintes temporelles ainsi qu'en fonction de l'espace dans lequel le processus se déroule : école, hôpital, prison, poste de police, services d'immigration ou juridiques, camps de réfugiés, etc.

Mais la divergence fondamentale se situe, pourrait-on dire, à un autre niveau : en mettant en rapport un professionnel et son client, l'interprète en milieu social participe à une relation asymétrique du point de vue du pouvoir et du savoir. Puisqu'il travaille à établir une communication à vocation réciproque, il doit s'investir dans une collaboration - en utilisant tantôt la diplomatie, tantôt l'invention ou même la ruse - qui consiste à créer un espace d'entente dans le but de satisfaire tous les actants du processus au-delà de la non-compréhension mutuelle ou des conflits parfois insurmontables qui risquent d’interrompre la circulation de la parole.

Bien que depuis une dizaine d'années la communautaire suscite un intérêt accru dans les milieux universitaires et professionnels, contrairement à la simultanée et à la consécutive, elle occupe toujours une place marginale dans les programmes de formation et de 
perfectionnement, et reste encore une profession non réglementée et peu reconnue. Pourtant, il s'agit d'une activité professionnelle largement pratiquée dans les sociétés multiethniques, activité qui ressemble en quelque sorte à un laboratoire qui rend possible l'observation des divers contextes de communication interculturelle, des divers comportements humains et institutionnels issus des divergences culturelles, sociales, économiques et juridiques qui, très souvent, risquent de ralentir, de dérailler ou même de bloquer l'échange verbal.

Le but de cet article est de cerner le processus interprétatif inscrit dans une dynamique d'emblée asymétrique et de nature « trialogique » puisqu'il s'agit de trois actants - le client, l'intervenant et l'interprète - qui participent à l'échange verbal tout en créant activement un espace de communication interculturel. Au-delà des questions techniques ou axées strictement sur la profession, il nous semble digne d'intérêt d'aborder la composante humaine qui intervient dans le processus interprétatif. C'est justement la communautaire qui représente un terrain propice à l'exploration des sentiments et des émotions qui, dans ce cas précis, font irruption et influent sur le déroulement de la communication. Si le transfert interlinguistique peut être facilement occulté dans bien des situations - et la traduction ethnocentrique en témoigne -, il ne peut en aucun cas être ignoré dans un face-à-face fondé sur une certaine réciprocité.

Comme le précise Daniel Gile, l'interprétation de conférence consiste à substituer un « discours de haut niveau formel et conceptuel dans une autre langue, dans son intégralité et en respectant le même niveau du discours » (Gile, 1995, p. 12). Dans ce cas, l'interprète ne traduit pas un simple dialogue mais un discours soutenu approprié à la situation de communication (conférence, assemblée, rencontre officielle, etc.). La même exigence est maintenue même lorsque le niveau de langue du locuteur est inapproprié : l'interprète aura instinctivement tendance à anticiper les attentes du public cible, autrement, à réduire la distance entre le locuteur et le récepteur en utilisant un langage plus soigné.

La communautaire, quant à elle, s'inscrit dans un lieu plus complexe : à cause de la hiérarchisation à plusieurs niveaux, elle met en scène une communication hautement asymétrique entre le professionnel et son client :

- langue mineure - langue majeure 
- savoir, compétence - non-savoir, ignorance

- institution - individu

- structures, lois, règlements - expérience, sentiments.

En règle générale, dans ce type d'intervention, les relations de pouvoir sont quasi palpables : si le professionnel s'investit dans la traduction (le décodage) de l'autre en tant qu'autorité dans son domaine d'activité, le client se sent constamment obligé de se traduire, c'est-à-dire de s'expliquer, de clarifier certaines informations, de se dire afin de se rendre accessible à son interlocuteur. Sujet hors de l'histoire, le client peut même apparaître comme une sorte de barbare s'exprimant dans une langue totalement incompréhensible et parfois incapable d'articuler un discours intelligible ou de répondre clairement à des questions qui lui sont posées. De telles situations sont particulièrement fréquentes lors des entretiens avec des illégaux, des ressortissants de pays aux régimes totalitaires ou aux prises avec des événements traumatisants (guerre, conflit militaire, génocide, etc.). L'utilisation des onomatopées, le bégaiement, les grimaces, la gesticulation prononcée, la posture, le mouvement du corps exagéré, sont souvent des signes extralinguistiques qui font voir l'autre en tant que différence radicale, celle qui échappe à la compréhension.

Il y a quelque chose de profondément gênant, de troublant même, dans la visibilité de l'étranger situé au centre de l'événement, coincé - le mot n'est pas trop fort - entre l'intervenant et l'interprète. C'est justement cette présence physique incontournable de l'autre (son corps, sa voix, ses émotions) qui détruit le fameux mythe de la transparence - condition utopique voulant éliminer les obstacles culturels et linguistiques au nom d'un sens universel transcendant les différences, et cela dans le but de faciliter la communication ou la circulation du savoir. Traditionnellement, la traduction abrite et génère plusieurs manifestations de ce mythe: la traduction cibliste ou ethnocentrique en serait une incarnation des plus répandues, parce qu'elle nivelle les différences en faveur de l'horizon d'attente du public cible. De même, d'autres types de traduction, comme le doublage qui élimine la présence de l'autre langue; l'interprétation simultanée qui éloigne l'interprète du champ de vision; la localisation qui vise une mobilité pure, sans obstacle ni frontière; ainsi que la traduction automatique qui écarte le sujet traduisant, tendent tous à occulter le passage, à effacer le transfert interlinguistique. 
D'un autre côté, autant la traduction sourcière que le sous-titrage et l'interprétation communautaire, introduisent des règles du jeu qui déconstruisent fondamentalement l'idée de la transparence, tout en mettant au défi la communication universelle. En d'autres termes, chaque fois que la traduction se manifeste - soit dans le respect de la lettre, soit par l'insertion des sous-titres, ou encore à cause de la présence réelle du sujet traduisant/interprétant -, une résistance l'accompagne que ce soit de la part du récepteur, du client ou d'un autre actant du processus.

C'est pourquoi la communautaire représente un cas à part, un cas intéressant à étudier étant donné que l'interprète assume pleinement $s a$ visibilité qui contribue aussi au succès de la communication ${ }^{1}$. Il est ce tiers (intermédiaire, médiateur, passeur) qui réduit la distance entre deux interlocuteurs - comme c'est d'ailleurs le cas dans la simultanée et la consécutive - et œuvre à créer un autre espace de communication en brisant la linéarité du transfert selon la ligne unidirectionnelle : émetteur - message - récepteur. L'interprète incarne alors une figure mobile dont la parole circule entre plusieurs axes dialogiques : intervenant - client (fidélité, neutralité), intervenant - interprète (commentaire), interprète client (commentaire), interprète - sa propre conscience (valeur ajoutée au contexte de la communication). En d'autres mots, son travail consiste à négocier le sens entre tous les participants du processus et non seulement à transmettre le contenu informationnel d'une langue à l'autre. Tantôt interprète (il transfère le sens), tantôt informateur (il commente, explique, ajoute de l'information), il peut aussi s'imposer en tant que juge ou témoin parlant au nom de quelqu'un ou en défendant une valeur (vérité, honnêteté, loyauté, solidarité, démocratie, etc.). Mais le juge ou témoin peut avoir une autre fonction encore : celle d'incorporer la parole de l'interlocuteur dans son propre discours et de se voir ainsi par le biais des opinions extérieures.

C'est le concept de tiers de Mikhaïl Bakhtine qui servira à mieux cerner le rôle de l'interprète et à suivre le chemin de la parole enfermée dans une cellule triangulaire, entre le professionnel, le client et l'interprète. Tout d'abord, le tiers remet en question l'épistémologie classique privilégiant le rapport d'identité entre le sujet et l'objet, comme si l'accès à la réalité pouvait se faire de manière directe.

\footnotetext{
${ }^{1}$ Les interprètes en milieu social travaillent également à distance (téléphone, conférence vidéo, etc.). Leur visibilité ou invisibilité varie donc d'un contexte de travail à l'autre.
} 
Confrontant les structures de pensée qui s'appuient sur l'analogie, la synthèse, le dualisme, la ressemblance, la non-contradiction, la continuité et l'identité, le tiers déconstruit la logique du même qui ne peut concevoir que deux types de relations : soit celle de fusion (tu es comme moi et inversement), soit celle d'opposition qui exige que l'autre reste dans un dehors absolu (tu n'es pas comme moi, comme nous, tu es donc exclu). Un autre point non négligeable à soulever : le tiers fait comprendre le travail de l'interprète au-delà de son rôle strictement professionnel, encadré par l'institution ${ }^{2}$. Cela permet donc d'attirer l'attention sur des facteurs humains qui interviennent lors de la communication interculturelle.

Par souci méthodologique, nous écartons d'emblée la première catégorie de tiers appelée "la position du troisième », que Bakhtine expose dans Problèmes du texte : elle est restreinte à la pensée abstraite, en dehors de la vie concrète :

il existe, dans l'abstrait, la position du troisième qu'on identifie à la "position objective» en tant que telle, à la «connaissance scientifique ». La position du troisième est entièrement justifiée là où un individu peut se mettre à la place d'un autre individu, là où les individus sont absolument interchangeables, ce qui est possible et fondé seulement dans le cas où l'on cherche une solution à des problèmes qui ne sollicitent pas la personne dans sa totalité et sa non-reproductibilité, autrement dit, là où l'homme se spécialise, exprimant seulement une partie détachée de son tout, de sa personne, là où sa qualité d' " ingénieur », de " physicien », etc., sera substituée à son moi. (Bakhtine, 1984, p. 364)

En linguistique pragmatique, cette catégorie de surdestinataire

joue un rôle d'un Autre qui serait à la fois un pair et un père, double idéal de l'énonciateur. Mais la relation à ce double variera en fonction de la position que l'on pense occuper dans le champ, du crédit que l'on pense y posséder en raison de sa trajectoire passée et de ses anticipations de carrière. Car l'article scientifique n'est pas un simple vecteur d'idées, il permet de conforter ou d'infléchir dans un certain sens la position que l'on occupe. (Maingueneau, 1991, p. 194)

\footnotetext{
2 « Role is a social science construct used to explain behaviour and examine attitudes between at least two participants in any social situation » (p. 30). Voir le chapitre 3, « The Role of the Interpreter », dans Adolfo Gentile, Uldis Ozolins et Mary Vasilakakos Liaison Interpreting. A Handbook, 1996, pp. 30-40.
} 
Du point de vue herméneutique, S. Fish explique l'importance de la communauté interprétative qui joue le rôle normatif dans la réception des textes. De même, la science a besoin d'une cohérence pour se légitimer. Comme le constate Lyotard, « [l]a vérité de l'énoncé et la compétence de l'énonciateur sont [...] soumises à l'assentiment de la collectivité des égaux en compétence » (Lyotard, 1979, p. 45). L'objectivité scientifique apparente est le fruit d'un travail critique qui véhicule aussi des valeurs extra-scientifiques considérables liées à la tradition critique. En traduction et en interprétation, ce tiers englobant se manifeste soit dans les normes qui régularisent l'activité professionnelle peu soucieuse du facteur humain et plutôt centrée sur la circulation efficace de l'information, soit sur les besoins ou les habitudes du récepteur. Contexte de communication vaste, éclaté et malléable, la communautaire force tous les participants à forger un modèle de communication polyphonique, ouvert à un autre temps et espace.

Outre ce tiers normatif dans lequel se dilue le subjectif et qui appartient à la pensée abstraite, au savoir objectivé, d'autres types de tiers sont définis par Bakhtine : ceux qui se manifestent dans le langage vivant où (1) le tiers assure soit l'écoute de tous les participants pour ensuite témoigner pour chacun d'eux (le tiers en tant que destinataire lointain), soit (2) une médiation dans le cas où le dialogue est interrompu (le tiers qui témoigne de la distance, du conflit), ou encore (3) un point de vue extérieur à la communication immédiate (le tiers en tant que juge et témoin).

Il nous semble que les trois catégories de tiers que nous venons de mentionner apportent un éclairage différent sur le processus interprétatif. Bien que le transfert reste une préoccupation majeure, les questions exclusivement linguistiques ou déontologiques risquent de voiler l'ensemble de la situation d'interprétation qui force à chaque situation l'ajustement de tous les participants. Si l'institution encadre le déroulement formel de l'intervention, en imposant aux trois parties (l'intervenant, le client et l'interprète) un code de conduite prédéterminé, comment saisir les moments critiques de la rencontre, moments qui font déraper la communication à cause du « surplus humain » incontrôlable? Quelle importance faut-il accorder à ce surplus? Comment le traiter? Comment le théoriser? ${ }^{3}$

${ }^{3}$ Bien que notre article soit à teneur théorique, la réflexion s'inscrit dans l'expérience professionnelle qui nous a amenée à travailler dans différents contextes : camp de réfugiés politiques en Italie, Consulat canadien à Rome, écoles québécoises, immigrations, etc. 
Tout d'abord, le tiers dans sa dimension humaine n'est pas une prédisposition donnée d'avance à tout individu : au contraire, il s'agirait plutôt d'un état de conscience profondément marqué par un éveil à l'égard de la différence. Bakhtine accorde cette position au personnaliste qui « se distingue [...] par sa liberté intérieure exceptionnelle, sa parfaite indépendance à l'égard du milieu ambiant » ${ }^{4}$ et pour qui l'existence et la connaissance se situent dans un espace mouvant constamment créé et recréé entre les interlocuteurs qui coexistent tous dans la conscience de celui ou de celle qui devient alors une personnalité. On pourrait croire que certains individus sont plus prédisposés à assumer le rôle d'un tiers : immigrants, exilés, membres d'une diaspora, etc. du fait d'appartenir à un univers hétérogène, fragmenté. Mais cela n'est pas pour autant garant d'ouverture d'esprit à l'égard de l'autre. Le tiers peut en effet être assumé par un individu qui vit en permanence dans sa communauté, mais qui résiste à la relation fusionnelle avec ses semblables. De même, l'interprète en milieu social manifeste différents comportements professionnels qui peuvent être motivés - au-delà des règles institutionnelles - par les sentiments ressentis à l'égard de l'étranger (peur, mépris, compassion, etc.) ou à l'égard de l'institution (accord ou désaccord).

1 - La première catégorie de tiers représente le pouvoir de création, la capacité de se projeter soit dans un temps, soit dans un lieu éloigné. Dans " Le problème du texte ", Bakhtine définit ainsi ce concept :
Un auteur ne peut jamais s'en remettre tout entier, et livrer toute sa production verbale à la seule volonté absolue et définitive de destinataires actuels ou proches (on sait que même les descendants les plus proches peuvent se tromper), et toujours il présuppose (avec une conscience plus ou moins grande) quelque instance de compréhension responsive qui peut être différée dans des directions variées. Tout dialogue se déroule, dirait-on, en présence du troisième, invisible, doté d'une compréhension responsive, et qui se situe au-dessus de tous les participants du dialogue (les partenaires) [...] (Bakhtine, 1984, pp. 336-337).

Bien que cette définition se limite au texte littéraire ou philosophique, ce tiers reste valide là où le discours s'ouvre à la différence, à un horizon inexploré, là où il crée des possibles. Incarnant le dépassement de soi, il oblige en effet à voir la situation à partir d'une perspective au-delà de

\footnotetext{
${ }^{4}$ Définition d'Askoldov, citée par Bakhtine, 1970, p. 39.
} 
l'immédiat. Tel est d'ailleurs le rôle de l'interprète en milieu social : dans bien des cas, il doit inventer les stratégies de communication, tout en sensibilisant les participants à des réalités inédites.

Variable, illimité, allant dans des directions variées, ce type de tiers présuppose une infinitude de possibilités imprévisibles. En littérature, par exemple, un lecteur contemporain d'une tragédie grecque devient son troisième participant engagé dans les événements décrits, mais distancé par rapport à eux, du fait d'appartenir à une autre époque et à un mode de pensée différent de celui de l'Antiquité. La communautaire, quant à elle, exige un effort soutenu pour surmonter les limites de la communication réciproque qui se déroule dans un cadre plus mobile et plus fragmenté que d'habitude à cause de la présence de plusieurs entités culturelles et linguistiques. Chaque pas en avant améliore réellement la communication et aide à mieux connaître l'autre. Dans certaines situations, l'interprète fournit de son propre gré le surplus d'information, soucieux d'apporter un éclairage plus vaste sur les phénomènes que l'intervenant ne saisit pas. Par exemple, si l'enfant russe qui vient d'arriver à Montréal au moment des changements politiques dans son pays manifeste un comportement d'enfant abusé, l'interprète peut attirer l'attention sur d'autres causes possibles que celle de l'autorité parentale pour aider les intervenants à mieux démêler certains troubles psychologiques, à mieux examiner les symptômes nommés et classés souvent trop rapidement selon les procédures en vigueur dans la société d'accueil. À long terme, le professionnel sera plus sensible, plus à l'écoute du vécu de son client. Et celui-ci, à son tour, ressentira instinctivement un effort de collaboration - et non seulement le poids du jugement - et sera encouragé à s'ouvrir davantage. Souvent, il s'agit de véritables moments magiques, surgissant sous forme de soupir de soulagement ou de rire convivial, qui font disparaître momentanément les barrières.

2 - Mais le tiers peut avoir une autre fonction : englober des voix distinctes, même celles qui sont antagonistes et qui s'excluent mutuellement. En même temps, ce tiers ne peut jamais se retrouver emprisonné dans un mot fermé, monologique (loi, ordre). Bakhtine explique cette situation comme "le contexte dialogique réel [...] accessible mais où nul contact de sens entre les répliques n'est possible (ou imaginable). Le degré zéro du rapport dialogique. C’est là qu'apparaît clairement le point de vue du troisième dans le dialogue (de celui qui ne participe pas au dialogue mais qui le comprend ). La compréhension du tout de l'énoncé est toujours dialogique. " (Ibid., 
p. 335). Et plus loin : « [c]omprendre c’est, nécessairement, devenir le troisième dans le dialogue » (Ibid., p. 335). Et la compréhension, vue de la position du tiers, de l'extériorité, a une dimension éthique, celle de la responsabilité d'être à l'écoute de l'autre ou de parler en son nom là où l'autre est réduit au silence ou au bégaiement.

Puisque ce tiers vise à forger un espace d'entente lors d'un conflit, il doit creuser un passage pour rejoindre l'autre par le biais d'une valeur humaine : responsabilité, compassion, projet commun, etc. C'est dans ce genre de situation que les moyens de communication habituels s'avèrent déficients ou inutiles, et c'est à l'interprète qu'incombe alors la responsabilité de lutter pour maintenir le dialogue. Au fond, le travail consiste à montrer que les différences ne sont qu'apparentes, illusoires et temporaires, et qu'il existe des points de jonction même au moment où un des interlocuteurs recule, hésite ou se réfugie dans le silence.

3 - La dernière catégorie de tiers est centrée sur l'interprète lui-même : à force de côtoyer la différence de très près, à cause de l'effort qu'il fournit pour l'entendre et la comprendre, l'interprète subit un travail sur sa propre conscience qui, pourrait-on dire, se fragilise à certains moments. Bakhtine explique la nature du juge et témoin dans ces mots : « un sur-homme, un sur-moi, autrement dit, un juge et témoin de tout l'homme (de tout le moi), par conséquent, non plus un homme, un moi, mais l'autre. Ma propre réfraction en un autre empirique au travers duquel il me faut passer pour déboucher sur le moi-pour-moi (ce moi-pour-moi peut-il être solitaire?). L’absolue liberté de ce moi. » (Ibid., « Les carnets », p. 357).

Déjà en 1923-24, Bakhtine aborde dans les «Notes de Poumpianskij » ce concept défini alors en termes de conscience religieuse ayant besoin d'une troisième présence, d'un juge potentiel qui évalue, parce que cette conscience est ouverte à une réévaluation de l'extérieur, étant donné qu'elle se trouve à l'opposé de la conscience morale, autonome, immanente et auto-consciente, qui n'exige que deux participants puisque le troisième est assimilé : un qui impose les principes moraux et l'autre qui lui obéit. Dans ce cas, l'évaluation de moi, conclut Bakhtine, provient toujours de l'extérieur (Bakhtine, 1992, p. 235), elle n'est pas intériorisée, incorporée dans le mot-pour-moi.

Plus tard, en étudiant l'œuvre de Dostoïevski, le penseur définit le phénomène du mot avec un " coup d'œil de côté » incarnant le discours sur soi-même, " déterminé par le mot réfléchi d'un "étranger”» 
(Bakhtine, 1970, p. 268). La plupart des héros dostoïevskiens vivent en effet en constante confrontation avec la présence, le regard et la parole de l'autre :

Le héros se juge en fonction de l'idée qu'il se fait d'autrui et de l'opinion sur soi qu'il lui suppose. La conscience de soi est sans cesse doublée par la conscience qu'en a autrui; le « moi pour moi-même » se réfère constamment au « moi pour les autres ». C'est pourquoi le mot du héros sur lui-même se construit sous l'influence incessante du mot d'autrui à son sujet. (Ibid., p. 269)

Il s'agirait d'un auditeur, ce que Dostoïevski souligne lui-même dans l'avant-propos à sa nouvelle Douce: "Tantôt l'homme se parle à lui-même, tantôt il s'adresse à quelque auditeur invisible, à un juge » ${ }^{5}$.

La signification de ce tiers loge dans l'obligation de prendre l'être humain dans sa dimension existentielle et morale et non psychologique ou instrumentale. Le juge et témoin est une autre conscience qui parle sans parler vraiment, qui s'exprime sans condamner, mais qui atteint les profondeurs de la conscience individuelle sur laquelle elle a le pouvoir d'agir. En d'autres termes, se situant à l'extérieur, sans coïncider avec le lieu de l'énonciation de l'autre/des autres, le juge et témoin présuppose l'impossibilité d'une compréhension totale, sans résidu, qui correspondrait en traduction et en interprétation à un transfert de sens total ou absolument fidèle. Par exemple, l'interprète qui travaille dans un camp de réfugiés politiques peut mettre en doute les valeurs démocratiques ou la validité des institutions qu'ils représentent : d'un côté, témoin de l'autorité absolue de l'État et de l'autre, de tant de situations critiques qui ont un impact immédiat et irréversible sur la vie des réfugiés, il risque de perdre sa neutralité, exposé à la présence tangible de l'autre, à son regard parfois suppliant, à sa peine, à son désespoir ou à sa colère.

Les trois catégories de tiers assument donc différentes fonctions : (1) s'ouvrir au discours de l'autre, (2) créer un espace de communication lors d'un conflit, (3) faire un travail sur sa propre conscience. Dans le domaine de la communautaire, l'interprète en tant que tiers fait parler les voix dans leur pluralité, leurs différences et leurs divergences, celles du client et de l'intervenant social qui se retrouvent dans un face-à-face. Le respect des actants consiste dans la préservation d'un univers plurivocal qu'ils représentent tous les deux, sans vouloir

${ }^{5}$ Cité par Bakhtine, 1984, p. 306, note 1. 
l'achever ni chercher à lui donner une unité cohérente. Coïncider avec le tiers signifie viser le dépassement des frontières, non pas pour englober, exclure ou condamner l'autre mais pour entrer en contact avec lui et dialoguer. Et dialoguer ne signifie pas créer un nous englobant, une somme totale des je, mais créer un lieu propice où tout le monde sera engagé dans la quête du sens. Le tiers ne présuppose pas non plus l'adhésion à une valeur quelconque : au contraire, c'est le positionnement, le point de vue qui compte, confronté par la réponse de l'interlocuteur.

Ce qui est justement propre à la communautaire c'est l'incertitude vis-à-vis du sens. Dans un face-à-face, la langue, certes, produit du sens, mais au-delà, le sensible s'impose et il faut le prendre en charge et l'interpréter. De plus, chaque rencontre crée un événement unique, imprévisible, parfois impossible à contrôler, et l'improvisation, la créativité, l'anticipation, sont de mise dans bien des cas.

La confrontation entre le professionnel et le client - qui représentent souvent des univers très différents - provoque en même temps l'effritement des certitudes, les déplacements constants des catégories vrai et faux. Comme l'interprète participe activement - et il n'a pas d'autre choix - au rapprochement, il remettra continuellement en question son propre travail, ses capacités, ses interventions, ses choix. Tout un savoir social sera également interrogé, et il sera souvent témoin des doutes que le professionnel subira vis-à-vis de l'institution qu'il représente. Tout à coup, les zones floues apparaissent parce que le sens ne peut plus advenir du seul fait d'appartenir à une société et d'être encadré par une institution. Ces moments sont parfois redoutables pour l'interprète qui doit s'ajuster à la matérialisation des conflits entre l'intervenant et son client (deux cultures, codes moraux) et parfois entre les compétences professionnelles de l'intervenant en milieu social et la vie qui les dépasse. Il n'est pas rare dans ces cas de sentir une certaine agressivité à l'égard du client : il est celui qui ébranle les certitudes, qui déstabilise la routine, qui remet en question l'état des choses. Parfois, cela provoque l'humiliation du client qui se voit totalement autre, inférieur, ignorant, jugé. Et plus tard, une fois le travail d'interprétation accompli, l'évaluation de l'interprète devient problématique à cause de la nature complexe de l'intervention : il est impossible de qualifier son travail de bon ou de mauvais; les catégories efficace/inefficace seraient probablement plus justes, mais là encore, il faudrait définir ces termes en fonction de chaque situation et des besoins ponctuels. 
Comme on l'a déjà dit, il est impensable de parler de la communautaire en fonction du transfert d'un contenu sémantique ou d'un message, comme si l'information circulait dans un conduit, passait par un canal unidirectionnel; le travail consiste plutôt à rendre la parole de l'autre - parole comme acte somatique qui sert à se dire, à se raconter dans toute sa visibilité et son audibilité -, travail dans lequel la conscience de l'interprète est totalement investie. Il lui est impossible de se séparer de cette parole qui devient relation ou appel, qui suscite engagement ou réaction. C'est d'autant plus évident lorsque l'interprète est placé devant une réalité dont le sens lui est encore étranger ou qui lui échappe. Il cherchera alors par anticipation le point de vue de la tierce personne pour trouver lui-même des réponses nécessaires. Puisque l'interprète interagit avec des institutions, son comportement proactif, centré sur l'anticipation, mènera à créer de nouvelles audiences, en déplaçant des frontières, en cherchant de nouveaux points de contact. C'est pourquoi, l'interprète est un point de jonction entre le réel et le virtuel, entre ce qu'on fait d'habitude ou ce qu'on doit faire selon les normes - et ce qu'on pourrait faire, créer, inventer, ouvrir.

Sans le tiers, le dialogue se limiterait à un accord absolu fusion, consensus - ou à deux monologues, à deux voix distinctes en pleine divergence, à deux individus atteints de surdité. Concevoir le tiers, au contraire, c'est affirmer la différence et contribuer à se rapprocher de l'autre sans l'anéantir. Et le rôle du tiers est particulièrement important dans les contextes asymétriques - comme c'est le cas de la communautaire - parce qu'il est le seul qui rend possible la traduction du discours marginal vers le discours dominant, sinon la place tout entière serait laissée à l'ordre du discours, au savoir doxique, qui n’a ni visage ni parole.

En fin de compte, l'interprétation en milieu social montre l'invalidité du modèle de communication classique (point d'origine point d'arrivée), mais qui est tout à fait valide en simultanée et en consécutive. Si cette activité reste dialogique, c'est parce que l'interprète peut influencer l'orateur et le déroulement du discours. Il participe activement à l'interaction linguistique et sociale en décontextualisant le message, en le ré-interprétant à partir de sa topologie, en donnant une deuxième version, parfois même élargie par ses commentaires additionnels. L'interprète non seulement traduit, mais coordonne la situation de l'interaction verbale : il questionne, explique, persuade, consent, réconforte, accuse, ment, nie, etc. C'est ainsi qu'un lieu d'échange complexe se réalise où, grâce à la présence du tiers, personne 
n'est réduit à l'objet; où les intentionnalités différentes se côtoient et où l'individu prend conscience d'appartenir à un monde plus vaste que son topos. Mais pour bâtir un tel lieu d'échange, il est nécessaire de créer un langage, une syntaxe, des modèles de communication capables d'établir des liens lors des interactions plurilingues asymétriques. Sans aucun doute, la communautaire occupe ce lieu d'échange difficile et souvent conflictuel qu'il n’est plus possible de négliger sous prétexte de fidélité et de transparence.

Le dernier point à souligner concerne les biographies des interprètes en milieu social. Antoine Berman, puisqu'il s'intéressait à l'herméneutique, a probablement été le premier à signaler l'importance des facteurs biographiques dans l'activité de traduction. Loin d'être uniforme du point de vue du comportement professionnel, le travail de l'interprète en milieu social variera selon son origine et selon son attitude vis-à-vis d'autres participants. L'analyse des dispositifs discursifs devrait donc inclure les points d'alliances selon l'origine de l'interprète :

- l'interprète en tant que membre de la société d'accueil (il sera porté à défendre les institutions et les valeurs de sa culture; son point d'alliance - le professionnel, l'institution, la société);

- en tant que compatriote du client (il risque de manipuler et de mentir au nom de la solidarité; son point d'alliance - le client);

- ni l'un ni l'autre, ce qui ne signifie pas pour autant la neutralité (selon son origine et l'origine du client, il risque de manifester de l'hostilité à l'égard du celui-ci s'il existe une injustice historique qui les sépare; son point d'alliance - soit le professionnel qui représente le pays d'accueil, soit la solidarité avec son pays d'origine).

Autant le tiers comme destinataire lointain, que le tiers qui intervient dans le dialogue au point zéro, ainsi que le tiers en tant qu'auditeur invisible (juge et témoin) - témoignent d'un surplus extra-verbal et extra-social qui agit sur l'intervention dans le milieu social, intervention d'emblée plurielle (langue, culture, mémoire, histoire). On a souvent identifié le tiers avec la force régulatrice qui serait exclusivement d'ordre social, imposant donc un certain comportement, des valeurs partagées par la communauté, alors que la pratique elle-même nous montre que la communautaire met en scène une interaction beaucoup plus complexe dans laquelle sont investies des forces et des motivations parfois peu perceptibles, parfois même obscures. 
Théoriser la communautaire sert justement à rendre cette pratique professionnelle un peu plus claire. Apparentée à la fois à la traduction et à l'interprétation, la communautaire constitue en même temps un nouveau défi. Tout d'abord, en tant que champ de réflexion émergeant, elle provoque des remaniements du domaine traductologique et remet en question un savoir déjà acquis; en tant que mode d'interprétation marginal, elle exige une recherche approfondie du processus lui-même encore peu étudié; en tant que pratique, elle soulève des questions d'ordre professionnel qui devraient définir la formation à suivre, le statut de l'interprète ainsi que l'éthique ${ }^{6}$. Si le scandale de la traduction - selon la formule de Venuti - est sa marginalisation généralisée, explorer sa propre périphérie et surtout ses propres blocages épistémologiques pourrait s'avérer hautement productif autant pour la traduction que l'interprétation, autant pour la théorie que la pratique.

\section{Université de Montréal}

\section{Références}

BAKHTINE, Mikhaïl (1970). Poétique de Dostoïevski. Tr. Isabelle Kolitcheff. Paris, Seuil.

- (1984). Esthétique de la création verbale. Tr. Alfreda Aucouturier. Paris, Gallimard.

- (1992). « Notes de Poumpianskij ». Bakhtine comme philosophe. Présenté par N. I. Nikolaev. Moscou, Naouka, pp. 221-252 (en russe).

FISH, Stanley (1980). Is There a Text in This Class? The Authority of Interpretative Communities. Cambridge et Londres, Harvard University Press.

\footnotetext{
${ }^{6}$ Nous signalons un excellent ouvrage collectif dans lequel les auteurs soulèvent justement ces questions: Translation, Research and Interpreting Research. Traditions, Gaps and Synergies, dir. Ch. Schäffner. Dans le chapitre 11, signé par F. Pöchnacker, on discute de l'importance qu'il faudrait accorder à la théorisation de la communautaire : " the need for an accepting attitude toward theoretical contributions, whether labeled as speculation, reflection, introspection, modeling, hypothesising or theorising, arises especially for IS as a young field rooted in and growing out of professional practice » (p.106).
} 
GENTILE, Adolfo, Uldis OZOLINS et Mary VASILAKAKOS (1996). Liaison Interpreting. A Handbook. Victoria (Australia), Melbourne University Press.

GILE, Daniel (1995). Regards sur la recherche en interprétation de conférence. Villeneuve d'Ascq, Presses Universitaires de Lille.

LYOTARD, François (1979). La condition postmoderne. Rapport sur le savoir. Paris, Les Éditions de Minuit.

MAINGUENEAU, Dominique (1991). L'analyse du discours. Introduction aux lectures de l'archive. Paris, Hachette.

SCHÄFFNER, Christina (2004). Translation, Research and Interpreting Research. Traditions, Gaps and Synergies. Clevedon, Buffalo, Toronto, Multilingual Matters Ltd.

RÉSUMÉ : L'interprétation communautaire : un modèle de communication " trialogique " - Il sera question de réfléchir sur l'interprétation communautaire, particulièrement sur les rapports qui existent entre le professionnel, le client et l'interprète lors d'un échange interculturel. Asymétrique, ce type d'intervention génère plusieurs niveaux de difficultés (langue, culture, code moral, pouvoir). En se servant du concept de tiers de Mikhaïl Bakhtine, il s'agira d'introduire une discussion sur les facteurs humains qui entrent en jeu lors d'une entrevue, au moment où l'interprète risque d'obéir à sa conscience ou à ses sentiments et non pas aux normes professionnelles et sociales.

ABSTRACT: Community Interpreting: A Trialogic Communication - This article explores some aspects of community interpreting, particularly the relationship between the professional, the client, and the interpreter, when participating in an intercultural exchange. Being asymetrical, this type of intervention generates many levels of difficulty (language, culture, moral code, power). Introducing Mikhail Bakhtin's concept of the third, we will discuss the human dimension of community interpreting, when the interpreter might obey his/her conscience and feelings rather than professional and social norms.

Mots-clés: interprétation communautaire, communication interculturelle, tiers, « trialogue ». 
Keywords: community interpreting, intercultural communication, the third, trialogue.

Aurelia Klimkiewicz : Département de linguistique et de traduction, Université de Montréal, C.P. 6128, succ. Centre-ville Montréal QC H3C 3J7.

Courriel : aurelia.klimkiewicz@umontreal.ca 